\title{
The Correlation between Rotational Gravity and Magnetic Field of Celestial Body
}

\author{
Ying-Qiu $\mathrm{Gu}^{*}$ \\ School of Mathematical Science, Fudan University, Shanghai 200433, China
}

The magnetic field of the earth plays an important role in the ecosystem, and the magnetic field of celestial bodies is also important in the formation of cosmic large-scale structures, but the origin and evolution of the celestial magnetic field is still an unresolved mystery. Many hypotheses to explain the origin have been proposed, but there are some insurmountable difficulties for each one. At present, the theory widely accepted in scientific society is the dynamo model, it says that, the movement of magnetofluid inside celestial bodies, which can overcome the Ohmic dissipative effect and generate persistent weak electric current and macroscopic magnetic field. However, this model needs an initial seed magnetic field, and the true values of many physical parameters inside the celestial body are difficult to obtain, and there is no stable solution to the large range of fluid motion. These are all difficulties for the dynamo model. Furthermore, it is difficult for the dynamo to explain the correlation between the dipole magnetic field and angular momentum of a celestial body. In this paper, by calculating the interaction between spin of particles and gravity of celestial body according to Clifford algebra, we find that a rotational celestial body provides a field $\Omega^{\alpha}$ for spins, which is similar to the magnetic field of a dipole, and the spins of charged particles within the celestial body are arranged along the flux line of $\Omega^{\alpha}$, then a macroscopic magnetic field is induced. The calculation shows that the strength of $\Omega^{\alpha}$ is proportional to the angular momentum of the celestial body, which explains the correlation between the magnetic intensity and angular momentum. The results of this paper suggest that further study of the effects of internal variables such as density, velocity, pressure and temperature of a celestial body on $\Omega^{\alpha}$ may provide some new insights into the origin and evolution of celestial magnetic field.

Keywords: Earth magnetic field, celestial magnetic field, magnetic dipole, Clifford algebra, Dirac equation, spin-gravity coupling potential, curved space-time.

The earth magnetic field is of great significance to the ecosystem. Geomagnetism has the function of navigation and location, and prevents the attack of solar wind against earth. The science of geomagnetism is developed from its practical applications. From the invention of the compass

*Electronic address: yqgu@fudan.edu.cn 
recorded by the Chinese in $250 \mathrm{BC}$ to the publication of the founding book on geomagnetism by the British doctor Gilbert in 1600, the science of geomagnetism has gone through an early stage of 1800 years. Queen Elizabeth's private doctor, Gilbert, was the first man to think systematically about geomagnetic phenomenon. He organized the scattered measurement data of geomagnetic field at different points under a unified framework, and concluded that the earth is a big magnet.

On the origin of geomagnetism, a hundred schools of thought contend, and more than a dozen different hypotheses have been put forward. However, there is no convincing explanation for the origin of geomagnetism, so it is listed by Einstein as one of the five major physics problems. Gilbert's hypothesis that the Earth is a permanent magnet, for example, faces a serious challenge to the Curie point temperature of the material: below the depth of 20 to 30 kilometers of the earth's crust, the temperature has exceeded the Curie point of most materials on the earth, so the material here cannot remain enough residual magnetism. The magnetism of the thin crustal material is far from enough to generate the observed geommagnetic field. Other hypotheses of geomagnetism origin, such as rotating magnetic effect, rotating charge effect, Hall effect, piezomagnetism effect and so on, are also denied due to the too small order of magnitude.

The widely accepted theory of the origin for the earth's magnetic field at present is the geodynamo. Its basic idea is that the conductive fluid of the outer core inside the earth is subjected to convective motion under the drive of various energy sources, and a magnetic field is generated by the current corresponding to the convection. That is, a process in which the driving energy is converted into the kinetic energy of the fluid, and then the kinetic energy is converted into the magnetic energy. If the converted magnetic energy can resist Ohmic dissipation, the magnetic field can be maintained by convective motion.

With the development of computer numerical technology, the dynamo model for the earth's magnetic field has been fully developed, and a large number of numerical simulations have been carried out. In references [1,2], the first three-dimensional self-consistent numerical solution of geomagneto-hydrodynamic equation with time is calculated. The equation describes the generation of thermal convection and magnetic field in a rapidly rotating spherical fluid shell with a solid conductive core.

As a rough simulation of the geodynamo, it is a self-sustaining supercritical generator, which maintains three magnetic field diffusion times for about 40000 years. The maximum velocity of the fluid in the outer core can reach $0.4 \mathrm{~cm} / \mathrm{s}$, and sometimes the magnetic field can reach 560 Gauss. Magnetic energy is usually 4000 times larger than the convective energy that maintains it. In this system in which the fluid interacts with the magnetic field, the toroidal magnetic field can 
be generated by the poloidal magnetic field(that is, the $\omega$ effect), and the poloidal magnetic field can also be generated by the toroidal magnetic field(that is, the $\alpha$ effect). A variety of dynamo models can be combined by these two basic effects.

Although great progress has been made in the numerical simulation of geodynamo, the origin of geommagnetic field is far from being solved, such as the origin of seed magnetic field, many properties and states of the earth's interior are also assumed, and the actual measurement is less. The inaccessibility of the earth core enables us to rely on the observation data in the vicinity of the surface and the space, and use the results of the laboratory simulation to infer and guess the state and process inside the earth.

Astronomical observation shows that the existence of large-scale regular magnetic field in rotating celestial bodies is a common phenomenon. In the solar system, the sun, Jupiter, Saturn, Uranus, Neptune and so on, all have strong dipolar moment magnetic fields. The magnetic fields of other distant stars, such as white dwarfs, pulsars and so on, are even greater[3, 4].

Even larger cosmic bodies, such as galaxy, galaxy cluster and so on, also have large-scale regular magnetic field distribution[5,6]. The origin of galactic magnetic field and extragalactic magnetic field is also one of the most challenging problems in modern Astrophysics. Magnetic fields are detected in all types of galaxy and cluster as long as appropriate observations are made. In addition, the magnetic field also exists in the distant galaxy with cosmic redshift. It is now widely accepted that the large-scale magnetic field in the disk galaxy is also amplified and maintained by the $\alpha \omega$-dynamo principle, in which the new magnetic field is regenerated through the combination of rotation and spiral turbulence. On the contrary, the intensity of magnetic fields in non-rotating or slow-rotating systems, such as elliptical systems and clusters, is relative much smaller.

But the galactic dynamo model itself is incomplete because it also does not explain the origin of the seed magnetic field used to start the dynamo. In addition, the time scale of magnetic field amplification in the standard $\alpha \omega$-dynamo model is too long to explain the magnetic field intensity observed in very young galaxies.

In , the theory of planet dynamo is used to discuss the Mars magnetic field. According to the theory, the key problems for the Mars magnetic field, such as dynamic mechanism, starting time and reason of stopping, are discussed in detail, and some key problems in the research are pointed out.

By analysis of observational data of the magnetic field for a large number of celestial bodies, it is found that the magnetic dipole moment of a celestial body has a strong correlation with its angular momentum, and the so-called Schuster-Wilson-Blackett relation approximately holds on a 
wide range of orders of magnitude $[4,7-10]$

$$
\frac{\mu}{L}=\frac{\beta \sqrt{G}}{2 c}
$$

in which $\mu, L$ are magnetic moment and angular momentum of the celestial body respectively, and $\beta \in \mathrm{O}(1)$ is a dimensionless number. The physical reason for this relationship was not specified at that time, so the result was not generally accepted.

In addition, in the analysis of [4], it is found that there is a significant positive correlation between $\log \mu$ and $\log L$ for cold stars. But such correlation between hot stars is much smaller. For the same kind of hot stars, $\log \mu$ and $\log L$ are even negatively correlated. In subsamples of the solar system, the correlation is basically the same as the slope of the cold star. On a large scale, $\log \mu$ and $\log L$ for different types of objects remain positively correlated(see Figure 9 in [4]).

The main theoretical explanations of Schuster-Wilson-Blackett relation are as follows: in [12], under Thoms-Fermi approximation the calculation shows that every ultra-dense material element in a gravitational field obtains a very small positive charge. But the celestial bodies as a whole are electrically neutral, because the negative charges are concentrated on their surface. In order of magnitude, the positive volume charge is very small (only $10^{-18} e$ ), but this is sufficient to explain the occurrence of the celestial magnetic field, as well as the existence of discrete spectra of the steady-state values of mass of stars and pulsars.

The explanation proposed in [10] is that, the function $\Phi_{l m}=\left(\eta / r_{g}\right) L^{j k} R_{j k l m}$ satisfies the Maxwell equation and can be used as a function to determine the electromagnetic properties of a rotating star, where $R_{j k l m}$ is the Riemann tensor, which determines the gravity field of the object, $r_{g}$ is the gravitational radius, and $\eta$ is a constant that must be determined by observation. The field $\Phi_{l m}$ describes the observed correlation of magnetic moments with angular momentum. It also explains the stability of the magnetic field of white dwarf and neutron stars under the Ohmic dissipation.

Among many suggestions on the origin of the earth's magnetic field in [8], a relatively satisfactory one is to make a slight modification to the law of electrodynamics. Electroneutral materials are considered to be a mixture of a large number of positive and negative charges, and their electrical and magnetic effects are usually balanced. If this balance is not precise, it will have small residual effects, including gravity and the Earth's magnetic field. Under this assumption, we can expect the moving matter to generate a magnetic field similar to the moving current, and we expect that there is a certain relationship between the magnetic field produced by the moving material and its gravitational action. In references [11], it is assumed that the moving mass can directly produce 
electromagnetic induction, so the rotating star is equivalent to a magnetic dipole.

Before expounding the author's point of view, let's review the concept of magnetic dipoles. The magnetic dipole model is a very small planar current-carrying coil. Its magnetic moment is defined as $\vec{\mu}=I \vec{S}, I$ is the current, $S$ is the coil loop area, and the direction of $\vec{S}$ has a right-hand spiral relationship with the current direction. The vector potential generated by the magnetic dipole is given by

$$
\vec{A}(\vec{r})=\frac{\mu_{0}}{4 \pi r^{2}}(\vec{\mu} \times \vec{r}), \quad\left(\mu_{0}=4 \pi \times 10^{-7}\left(\mathrm{~N} / \mathrm{A}^{2}\right)\right),
$$

$\mu_{0}$ is vacuum permeability, $\vec{r}$ is the position vector from the center of the dipole to the measuring point. The magnetic field intensity of the magnetic dipole is calculated by

$$
\vec{B}=\nabla \times \vec{A}=\frac{\mu_{0}}{4 \pi r^{3}}(3(\vec{\mu} \cdot \hat{r}) \hat{r}-\vec{\mu})+\frac{2 \mu_{0}}{3} \vec{\mu} \delta^{3}(\vec{r}),
$$

in which $\delta^{3}(\vec{r})$ is the Dirac- $\delta$. Since the Dirac- $\delta=0$ for any $\vec{r} \neq 0$, the Dirac- $\delta$ vanishes when computing the field strength in the region $r>0$. However, in quantum mechanics at the atomic scale, this term will make an important contribution. The Dirac- $\delta$ function of the dipolar magnetic field causes the atomic energy level to split, thus forming the hyperfine structure. In astronomy, the hyperfine structure of hydrogen atoms gives a $21 \mathrm{~cm}$ spectra, which is the broadest electromagnetic radiation in the universe except $3 \mathrm{~K}$ background radiation in the radio wave range of electromagnetic radiation. In the spherical coordinate system, the magnetic flux equation of (3) is as follows

$$
\frac{d \vec{r}}{d s}=\vec{B} \Rightarrow \frac{d r}{d \theta}=\frac{2 r \cos \theta}{\sin \theta} \Leftrightarrow r=R \sin ^{2} \theta
$$

When there are multiple magnetic dipoles, according to the superposition principle, the total magnetic field is the total vector sum of the magnetic field of each magnetic dipole. In order to calculate the magnetic moment and magnetic field of a planet, according to the structure of the planet, the ring or sphere can be selected as the magnetic dipole element, and then the whole magnetic moment and magnetic field of the planet can be obtained by integral. The distribution of magnetic fields outside the planet is very close to that produced by a single magnetic dipole.

We know that the properties of electrons and protons are described by quantum mechanics, so strictly investigating the interaction between Dirac spinor and gravitational field may be the key to unravel the secrets of celestial magnetic field. Denote the element of space-time by

$$
d \mathbf{x}=\gamma_{\mu} d x^{\mu}=\gamma^{\mu} d x_{\mu}=\gamma_{a} \delta X^{a}=\gamma^{a} \delta X_{a}
$$

In which the tetrad satisfies Clifford algebra $C \ell(1,3)$

$$
\gamma_{a} \gamma_{b}+\gamma_{b} \gamma_{a}=2 \eta_{a b}, \quad \gamma_{\mu} \gamma_{\nu}+\gamma_{\nu} \gamma_{\mu}=2 g_{\mu \nu}
$$


where $\eta=\operatorname{diag}(1,-1,-1,-1)$ is Lorentz metric. The relation between the tetrad coefficient and the metric is given by

$$
\begin{gathered}
\gamma^{\mu}=h_{a}^{\mu} \gamma^{a}, \quad \gamma_{\mu}=l_{\mu}^{a} \gamma_{a}, \quad l_{\mu}^{a} h_{b}^{\mu}=\delta_{b}^{a}, \quad l_{\mu}^{a} h_{a}^{\nu}=\delta_{\mu}^{\nu} . \\
h_{a}^{\mu} h_{b}^{\nu} \eta^{a b}=g^{\mu \nu}, \quad l_{\mu}^{a} l_{\nu}{ }_{\nu} \eta_{a b}=g_{\mu \nu} .
\end{gathered}
$$

In [13], we derived the Dirac equation in curved space-time in detail. In the natural unit system, we have

$$
\alpha^{\mu} \hat{p}_{\mu} \phi+s_{\mu} \Omega^{\mu} \phi=m \gamma^{0} \phi
$$

where $\alpha^{\mu}$ is current operator, $\hat{p}_{\mu}$ is momentum operator and $s_{\mu}$ spin operator. They defined respectively as

$$
\alpha^{\mu}=\operatorname{diag}\left(\varrho^{\mu}, \widetilde{\varrho}^{\mu}\right), \quad \hat{p}_{\mu}=i\left(\partial_{\mu}+\Upsilon_{\mu}\right)-e A_{\mu}, \quad s^{\mu}=\frac{1}{2} \operatorname{diag}\left(\varrho^{\mu},-\widetilde{\varrho}^{\mu}\right),
$$

where $\varrho^{\mu}=h^{\mu}{ }_{a} \sigma^{a}, \widetilde{\varrho}^{\mu}=h_{a}^{\mu} \widetilde{\sigma}^{a}$ is the Pauli matrices in curved space-time. We call $\Upsilon_{\mu}$ Keller connection. Professor Jaime Keller was the late former editor in chief of 'Advances in Applied Clifford Algebras'. He founded this journal and made outstanding contributions to promote the research and application of Clifford algebra. $\Omega_{\mu}$ is a new pseudo vector describing the interaction between spinor spin and gravity, and they are defined by

$$
\Upsilon_{\mu}=\frac{1}{2} h_{a}^{\nu}\left(\partial_{\mu} l_{\nu}^{a}-\partial_{\nu} l_{\mu}^{a}\right), \quad \Omega^{\alpha}=\frac{1}{4} \epsilon^{d a b c} h_{d}^{\alpha} h_{a}^{\beta} S_{b c}^{\mu \nu} \partial_{\beta} g_{\mu \nu}, \quad S_{a b}^{\mu \nu} \equiv-h_{[a}^{\{\mu} h_{b]}^{\nu\}} .
$$

In the Hamiltonian of a spinor we derived a spin-gravity coupling potential $s_{\mu} \Omega^{\mu}$. If the metric can be orthogonalized, we have $\Omega_{\mu} \equiv 0$, and then the spin and gravity are decoupled.

If the gravitational field is generated by a rotating ball, the corresponding metric, like the Kerr metric, cannot be diagonalized. At this time, the spin-gravity coupling term have non-zero coupling effect. Similarly to the case of charged particles in a magnetic field, the spins of spinors will be automatically arranged along the fluxes of $\Omega_{\mu}$. If the spins of all charged particles are arranged regularly along these fluxes, a macroscopic magnetic field will be induced. In order to clarify whether this magnetic field is related to the magnetic field of celestial bodies, we examine the flux of $\Omega_{\mu}$ field of a rotating star. The metric produced by the rotating sphere is similar to the Kerr metric, and in the asymptotically flat space-time we have the line element in quasi-spherical coordinate system[14]

$$
\begin{gathered}
d \mathbf{x}=\gamma_{0} \sqrt{U}(d t+W d \varphi)+\sqrt{V}\left(\gamma_{1} d r+\gamma_{2} r d \theta\right)+\gamma_{3} \sqrt{U^{-1}} r \sin \theta d \varphi \\
d \mathbf{x}^{2}=U(d t+W d \varphi)^{2}-V\left(d r^{2}+r^{2} d \theta^{2}\right)-U^{-1} r^{2} \sin ^{2} \theta d \varphi^{2}
\end{gathered}
$$


in which $(U, V, W)$ is just functions of $(r, \theta)$. As $r \rightarrow \infty$ we have

$$
U \rightarrow 1-\frac{2 m}{r}, \quad W \rightarrow \frac{4 L}{r} \sin ^{2} \theta, \quad V \rightarrow 1+\frac{2 m}{r},
$$

where $(m, L)$ are mass and angular momentum of the star respectively. For common stars and planets we always have $r \gg m \gg L$. For example, we have $m \doteq 3 \mathrm{~km}$ for the sun. The nonzero tetrad coefficients of metric (13) are given by

$$
\left\{\begin{array}{l}
l_{t}^{0}=\sqrt{U}, l_{r}{ }^{1}=\sqrt{V}, l_{\theta}{ }^{2}=r \sqrt{V}, l_{\varphi}^{3}=\frac{r \sin \theta}{\sqrt{U}}, l_{\varphi}^{0}=\sqrt{U} W, \\
h_{0}^{t}=\frac{1}{\sqrt{U}}, h^{r}{ }_{1}=\frac{1}{\sqrt{V}}, h^{\theta}{ }_{2}=\frac{1}{r \sqrt{V}}, h^{\varphi}{ }_{3}=\frac{\sqrt{U}}{r \sin \theta}, h^{t}{ }_{3}=\frac{-\sqrt{U} W}{r \sin \theta} .
\end{array}\right.
$$

Substituting it into (11) we get

$$
\begin{aligned}
\Omega^{\alpha} & =h_{0}^{t} h_{1}^{r} h_{2}^{\theta} h_{3}^{\varphi}\left(0, \partial_{\theta} g_{t \varphi},-\partial_{r} g_{t \varphi}, 0\right) \\
& =\left(V r^{2} \sin \theta\right)^{-1}\left(0, \partial_{\theta}(U W),-\partial_{r}(U W), 0\right) \\
& \rightarrow \frac{4 L}{r^{4}}(0,2 r \cos \theta, \sin \theta, 0) .
\end{aligned}
$$

By (16) we find that, the intensity of $\Omega^{\alpha}$ is proportional to the angular momentum of the star, that is to say, the absolute value of the spin-gravitational coupling potential of charged particles is proportional to the angular momentum of the star.

Now we check the flux line of $\Omega^{\alpha}$. By (16) we have

$$
\frac{d x^{\mu}}{d s}=\Omega^{\mu} \Rightarrow \frac{d r}{d \theta}=\frac{2 r \cos \theta}{\sin \theta} \Leftrightarrow r=R \sin ^{2} \theta .
$$

(17) shows that, the flux lines of $\Omega^{\alpha}$ and the magnetic fluxes (4) of the magnetic dipole (3) coincide with each other. According to the above conclusions, we know that the spin-gravity coupling potential of charged particles will certainly induce a macroscopic dipolar magnetic field for the star, and it should be approximately in accordance with the Schuster-Wilson-Blackett relation (1).

So far, we have two more questions to explain for the magnetic fields of the star and planet: The first one is how to understand that, the direction of the magnetic dipole of a planet always deviates a little from the direction of angular momentum? The second is how to understand the negative correlation between the magnetic dipoles and angular momentum of the same type of hot stars (see figure 6, 7, 8 in [4]). In the above discussion, we only consider a simplified model with concentrated parameters, that is, only the total mass $m$ and total angular momentum $L$ of the star are considered, but the distribution of variables such as mass density, temperature, and velocity are ignored. These factors obviously have significant influences on the intensity and distribution of magnetic field of a star. For example, temperature reflects the speed of particle motion, and high 
temperature will inevitably reduce the order of spin arrangement, and then reduce the magnetic dipole intensity of a star, so the magnetic field of the star will be relatively weakened with the increase of temperature. So a more detailed study should include physical variables inside the star such as mass density, speed of fluid, temperature, and pressure. By introducing these parameters, we will get a more accurate model for the magnetic field of celestial body.

Furthermore, the metric of a rotating celestial body is non-diagonal, which will produce some dynamic effect. The precession of the planet magnetic dipole relative to the rotational pole should be a relativity effect, so in order to clarify these effect we need more detailed dynamic analysis. After considering these factors, we may be able to answer the above two questions.

The origin and evolution of celestial magnetic field is a complex and difficult problem. Compared with the existing hypotheses and theories, the explanation proposed in this paper seems to be more natural and reasonable, and may be closer to the deep essence. The rotating planet provides a weak gravitational field for particle spin like the magnetic dipole magnetic field, which is a somewhat unexpected discovery, and one has to admire the magic and subtlety of creation. As Professor James Nester encouraged me when I studied the decomposition of spinor connections (11), "There may be a deep wisdom that we haven't appreciated". The spin-gravity coupling potential is equivalent to equip each particle with a pair of eyes of navigation and location functions. The results of this paper suggest that further study of the effects of internal parameters such as density, velocity, pressure and temperature of a celestial body on $\Omega^{\alpha}$ field may play an important role in further understanding the magnetic field of celestial bodies. But this is also a complex systematic project, which requires the joint efforts of multi-disciplinary colleagues.

[1] Glatzmaier G A , Roberts P H . A three-dimensional convective dynamo solution with rotating and finitely conducting inner core and mantle[J]. Physics of the Earth and Planetary Interiors, 1995, 91(1$3): 0-75$.

[2] Glatzmaier G A , Roberts P H . Simulating the geodynamo[J]. Contemporary Physics, 1997, 38(4):269288.

[3] Ahluwalia D V , Wu T Y . On the magnetic field of cosmological bodies[J]. Lett. Nuovo Cimento (1978) 23: 406. https://doi.org/10.1007/BF02786999

[4] Arge C N, Mullan D J, Dolginov A Z . Magnetic moments and angular momenta of stars and planets[J]. The Astrophysical Journal, 1995, 443(2):795-803.

[5] L. M. Widrow, Origin of Galactic and Extragalactic Magnetic Fields, Rev. Mod. Phys. 74 (2002) 775;

[6] P. P. Kronberg, Extragalactic Magnetic Fields, Rept. Prog. Phys. 57 (1994) 325. 
[7] A. Schuster, A critical examination of the possible causes of terrestrial magnetism, Proc. Phys. Soc. London 24, 121 (1911)

[8] H. A. Wilson, An experiment on the origin of the earth's magnetic field, Proc. R. Soc. 104, 451 (1923).

[9] P.M.S. Blackett, The magnetic field of massive rotating bodies, Nature 159, 658-666 (1947)

[10] Dolginov A., Electromagnetic Field Created by Rotation of Celestial Bodies[J]. Journal of Modern Physics, 2016, 7(16):2418-2425.

[11] Jacob Biemond,The magnetic field of pulsars and the gravito-magnetic theory,In: Trends in Pulsar Research (Ed. Lowry, J. A.), Nova Science Publishers, New York, Chapter 2 (2007). arXiv:astro$\mathrm{ph} / 0401468$

[12] B.V. Vasiliev, Gyro-Magnetic Relations and Masses of Stars,arXiv:astro-ph/0002048

[13] Ying-Qiu Gu, Space-Time Geometry and Some Applications of Clifford Algebra in Physics[J]. Advances in Applied Clifford Algebras, 2018, 28(4):79.

[14] Ying-Qiu Gu, The Series Solution to the Metric of Stationary Vacuum with Axisymmetry[J]. Chinese Physics B, 2008, 19(3):90-100. 